\title{
Association of Dysphagia With Supratentorial Lesions in Patients With Middle Cerebral Artery Stroke
}

\author{
Bo-Ram Kim, MD, PhD ${ }^{1}$, Won-Jin Moon, MD, $\mathrm{PhD}^{2}$, Hyuntae Kim, $\mathrm{MD}^{1}$, \\ Eunhwa Jung, $\mathrm{OT}^{1}$, Jongmin Lee, $\mathrm{MD}, \mathrm{PhD}^{1}$
}

Departments of ${ }^{1}$ Rehabilitation Medicine and ${ }^{2}$ Radiology, Konkuk University School of Medicine \& Konkuk University Medical Center, Seoul, Korea

\begin{abstract}
Objective To determine the supratentorial area associated with poststroke dysphagia, we assessed the diffusion tensor images (DTI) in subacute stroke patients with supratentorial lesions.

Methods We included 31 patients with a first episode of infarction in the middle cerebral artery territory. Each subject underwent brain DTI as well as a videofluoroscopic swallowing study (VFSS) and patients divided were into the dysphagia and non-dysphagia groups. Clinical dysphagia scale (CDS) scores were compared between the two groups. The corticospinal tract volume (TV), fractional anisotropy (FA) and apparent diffusion coefficient (ADC) values were calculated for 11 regions of interest in the supratentorial area-primary motor cortex, primary somatosensory cortex, supplementary motor cortex, anterior cingulate cortex, orbitofrontal cortex, parietooccipital cortex, insular cortex, posterior limb of the internal capsule, thalamus, and basal ganglia (putamen and caudate nucleus). DTI parameters were compared between the two groups.

Results Among the 31 subjects, 17 were diagnosed with dysphagia by VFSS. Mean TVs were similar across the two groups. Significant inter-group differences were observed in two DTI values: the FA value in the contra-lesional primary motor cortex and the ADC value in the bilateral posterior limbs of the internal capsule (all $\mathrm{p}<0.05)$.

Conclusion The FA value in the primary motor cortex on the contra-lesional side and the ADC value in the bilateral PLIC can be associated with dysphagia in middle cerebral artery stroke.
\end{abstract}

Keywords Diffusion tensor imaging (DTI), Deglutition disorders, Middle cerebral artery, Stroke

\footnotetext{
Received September 11, 2015; Accepted December 16, 2015

Corresponding author: Jongmin Lee

Department of Rehabilitation Medicine, Konkuk University Medical Center, 120-1 Neungdong-ro, Gwangjin-gu, Seoul 05030, Korea. Tel: +82-2-20305351, Fax: +82-2-2030-5379, E-mail: leej@kuh.ac.kr

ORCID: Bo-Ram Kim (http://orcid.org/0000-0002-5463-1268); Won-Jin Moon (http://orcid.org/0000-0002-8925-7376); Hyuntae Kim (http://orcid. org/0000-0001-9230-9977); Eunhwa Jung (http://orcid.org/0000-0003-4331-7369); Jongmin Lee (http://orcid.org/0000-0001-8718-0099).
}

(c) This is an open-access article distributed under the terms of the Creative Commons Attribution Non-Commercial License (http://creativecommons.org/ licenses/by-nc/4.0) which permits unrestricted noncommercial use, distribution, and reproduction in any medium, provided the original work is properly cited. Copyright $\odot 2016$ by Korean Academy of Rehabilitation Medicine 


\section{INTRODUCTION}

Swallowing is a complex physiological process involving voluntary and reflexive motor activity, sensorimotor integration, salivation, and visceral regulation [1]. Swallowing abnormalities are a common functional impairment after acute stroke, affecting up to $50 \%$ of the patients [2]. Post-stroke dysphagia may cause severe complications, such as aspiration pneumonia, malnutrition, dehydration, and fatal outcomes [3]. Despite the clinical importance of swallowing, neither an anatomical region nor the pathophysiology of dysphagia has been clearly identified. Thus, much effort has been made to elucidate the regions associated with swallowing function and to evaluate the factors affecting long-term outcomes of dysphagia. Although the medulla is known to have a role in dysphagia, there is insufficient information about the supratentorial region involved in swallowing. Gonzalez-Fernandez et al. [4] reported that the primary somatosensory, motor, and motor supplementary areas, orbitofrontal cortex, basal ganglia, and internal capsule are associated with dysphagia in acute supratentorial ischemic stroke patients.

Diffusion tensor imaging (DTI) is a well-established magnetic resonance imaging (MRI) method that measures both the integrity and direction of tracts using anisotropic diffusion; i.e., diffusion of water molecules along the axonal tracts within high density white-matter nerve fibers [5]. It provides valuable information about the microstructural changes in the tissue by applying a motion probing gradient in some directions for in vivo monitoring of potentially-restricted water molecules [6].

The two most commonly used indices of water diffusion in the tissue are the apparent diffusion coefficient (ADC) and the directional dependence of diffusion (diffusion anisotropy), expressed as fractional anisotropy (FA) [7]. ADC measures the general diffusivity of the water in brain tissue; brighter regions indicate the areas where water molecules diffuse faster than in darker regions. ADC values increase as a result of accumulation of intracellular or extracellular water, as well as when cells proliferate [8]. FA describes the degree of functional anisotropy of the tissue, which represents diffusivity using the orientation of restricting barriers formed by glia and has been used to indicate white matter tracts $[8,9]$. FA decreases with disruption of fiber tracts, i.e., demyelization or loss of axonal integrity $[9,10]$ and increases with axonal sprout- ing [11]. Therefore, ADC and FA may help to understand cellular and axonal remodeling in the post-stroke setting.

We speculated that there may be lesional differences in patients with dysphagia, since swallowing is a complex process involving not only the brainstem, but also the cerebral cortex. In this study, we investigated the DTI parameters, such as corticospinal tract volume (TV), FA, and ADC, in subacute stroke patients with supratentorial lesions. These data were used to determine the supratentorial regions associated with post-stroke dysphagia, and to verify the microstructural changes that cannot be found on conventional brain MRI.

\section{MATERIALS AND METHODS}

\section{Subjects}

A retrospective chart review was performed at Konkuk University Medical Center. From March 2008 to June 2012, a total of 155 stroke patients underwent a videofluoroscopic swallowing study (VFSS). Only those with middle cerebral artery (MCA) lesions were included in the current study. Exclusion criteria were (1) subarachnoid hemorrhage; (2) previous stroke or swallowing disorders; (3) concomitant lesions in the brainstem or cerebellum; (4) other diseases that lead to dysphagia, such as multiple sclerosis, oropharyngeal structural damage, dementia [12], or neurodegenerative disorders [13,14]; (5) decreased consciousness, poor cooperation for VFSS, or inability to maintain a sitting position for VFSS; (6) contraindication for MRI; or (7) refusal of DTI. The final sample included 31 MCA stroke patients who had undergone DTI within 6 weeks of a stroke. The Korean version of the Western Aphasia Battery [15] was used to detect aphasia, and the line bisection test [16] and Albert test [17] were used to detect hemispatial neglect. The study was approved by the Institutional Review Board at Konkuk University Medical Center (No. KUH1180010).

\section{Swallowing function test}

VFSS, clinical dysphagia scale (CDS), and diet level were assessed on admission. VFSS was performed by an experienced rehabilitation physician, assisted by an occupational therapist and a radiologic technician in the fluoroscopic laboratory. Subjects were seated upright in a wheelchair, and the fluoroscopic X-ray tube was focused in a lateral plane and the placement was verified. 
Each subject swallowed two $5 \mathrm{~mL}$ and then two $10 \mathrm{~mL}$ dysphagia diet boluses (mixture of Solotop-HD, barium sulfate powder for suspension). Dysphagia diet level was based on the study by Han et al. [18], which suggested that dysphagia diet should be divided into four classes based on the level of viscosity with reference values of $1,000,100$, and $10 \mathrm{cP}$ by a viscometer and 1,3 , and $4 \mathrm{~cm}$ by the line spread test. In the present study, we used the line spread test for dysphagia diet for VFSS. Dysphagia diet levels were defined as follows. Level $1,<1 \mathrm{~cm}$ by the line spread test, $3 \mathrm{~g}$ of thickener (Toromi-up; Nisshin OilliO, Tokyo, Japan) added to $100 \mathrm{~mL}$ of water; level 2, $1-3 \mathrm{~cm}$ by the line spread test, $2 \mathrm{~g}$ of thickener (Toromiup) added to $100 \mathrm{~mL}$ of water; level 3, 3-4 $\mathrm{cm}$ by the line spread test, $1 \mathrm{~g}$ of thickener (Toromi-up) added to 100 $\mathrm{mL}$ of water; level $4,>4 \mathrm{~cm}$ by the line spread test, $0.7 \mathrm{~g}$ of thickener (Toromi-up) added to $100 \mathrm{~mL}$ of water; level 5 , water. Lip closure, bolus formation, mastication, and oral transit time were measured in the oral phase. Normal oral transit time was defined as less than 1 second. In the pharyngeal phase, laryngeal elevation, cricopharyngeal dysfunction, oronasal regurgitation, residues in the valleculum and pyriform sinus, pharyngeal transit time, and aspiration status were observed. Residues in the valleculum and pyriform sinus less than $10 \%$ were considered normal; normal pharyngeal transit time was defined as less than 1 second. Aspiration was defined when the bolus passed through the vocal folds. Furthermore, in the esophageal phase, the esophageal relaxation status was examined. The results of the initial examination were then reviewed, and dysphagia was confirmed if abnormal findings were apparent during any one of the oral, pharyngeal, or esophageal phases. Subjects were divided into two groups according to the VFSS findings (dysphagia group and non-dysphagia group).

The CDS (Appendix 1) was used to quantify the clinical severity of dysphagia. The CDS has been confirmed as a quantitative clinical tool that reflects the VFSS findings well, and it can be adapted to various patients with dysphagia irrespective of the causal disorders [19].

\section{DTI data acquisition}

All imaging was performed on a 3.0-Tesla MRI system (Signa HDxt; GE Medical System, Milwaukee, WI, USA) with a standard 8-channel phase array head coil. For each of the 15 noncollinear and noncoplanar diffusion- sensitizing gradients, approximately 70 contiguous slices parallel to the anterior commissure-posterior commissure line were acquired. Imaging parameters were as follows: matrix, $120 \times 120$ matrix; field of view, $240 \times 240 \mathrm{~mm}^{2}$, TE, $84 \mathrm{~ms}$; TR, 16,000 ms; $\mathrm{b}=800 \mathrm{~mm}^{2} / \mathrm{s}$; and slice thickness, $2 \mathrm{~mm}$. In addition to DTI, conventional T2-FLAIR images were obtained.

\section{DTI analysis}

Raw image data were transferred in the DICOM format. All DTI images were corrected for eddy current-induced image distortions using FSL software (The Analysis Group, FMRIB, Oxford, UK; http://www.fmrib.ox.ac.uk/ fsl). DTI analyses were performed by an experienced rehabilitation medicine physician using the DTI studio (https://www.mristudio.org). All parameters were measured twice, and results were averaged. To reconstruct the corticospinal tract (CST), the first ROI was drawn free-hand in the posterior limb of the internal capsule, through which motor fibers descend; the second ROI was drawn in the basis pontis of the lower pons, using the "AND" operation $[20,21]$. The CST volume was calculated by the number of voxels in the reconstructed CST.

FA and ADC values were measured for manually-identified and atlas-based 11 ROIs (Fig. 1). These ROIs were defined after a literature review to identify the areas that have been implicated in swallowing function. We chose to include the areas whose function is unclear or whose involvement in swallowing has been questioned, in addition to the areas that have already been implicated in swallowing function. Polygonal ROIs were drawn on the slice of the color map or FA map, according to the previously defined criteria.

To standardize and unify the boundaries of the ROIs, two raters devised the training sessions. The ROIs were drawn after obtaining consensus about the boundaries of ROIs using a few selected images. The first author (rater A) manually investigated the ROIs, and 10 cases were randomly selected to determine the inter-rater reliability of the measurements. An experienced neuroradiologist, with an extensive background in neuroanatomy, participated as the other rater (rater B) and conducted the same drawing process while also being blinded to the diagnosis, gender, age and severity of stroke.

Intraclass correlation coefficients were calculated for the primary somatosensory cortex (PSC), primary mo- 

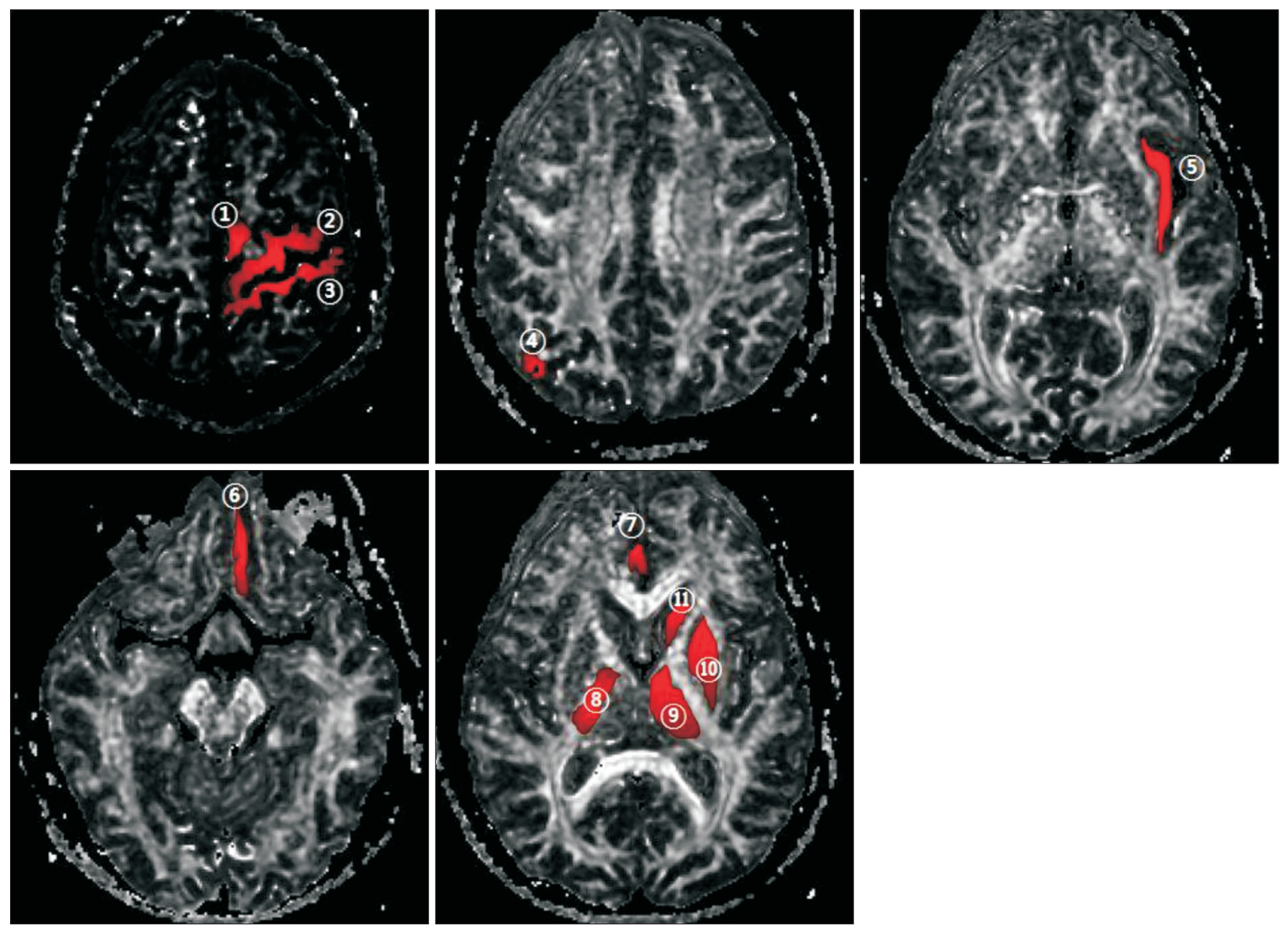

Fig. 1. The eleven regions of interest used in the study overlaid on fractional anisotropy maps: (1) supplementary motor cortex, (2) primary motor cortex, (3) primary somatosensory cortex, (4) parieto-occipital cortex, (5) insular cortex, (6) orbitofrontal cortex, (7) anterior cingulate cortex, (8) posterior limb of internal capsule, (9) thalamus, (10) basal ganglia (globus pallidus and putamen), and (11) basal ganglia (caudate nucleus).

tor cortex (PMC), supplementary motor cortex (SMC), anterior cingulate cortex (ACC), orbitofrontal cortex (OFC), parieto-occipital cortex (POC), insular cortex (IC), posterior limb of the internal capsule (PLIC), thalamus, and basal ganglia, from a subset of 10 cases. Although the sample size was small, there was good inter-rater reliability. For the ROIs, the intraclass correlation coefficients were 0.973 (95\% confidence interval [CI], 0.90-0.99) for the PSC, 0.96 (95\% CI, 0.84-0.99) for the PMC, 0.65 (95\% CI, 0.71-0.90) for the SMC, 0.90 (95\% CI, 0.67-0.98) for the ACC, 0.58 (95\% CI, 0.29-0.88) for the OFC, 0.93 (95\% CI, 0.74-0.98) for the POC, 0.89 (95\% CI, 0.62-0.97) for the IC, 0.93 ( $95 \%$ CI, 0.75-0.98) for the PLIC, 0.67 (95\% CI, $0.12-0.91$ ) for the thalamus, and 0.75 (95\% CI, 0.28-0.93) for the basal ganglia. The measurements obtained by rater A were used as the final measured value.

DTI parameters were compared between the two groups and were assessed for correlation with clinical dysphagia parameters.

\section{Statistical analysis}

For comparison between the dysphagia and non-dysphagia groups, clinical factors, including age, duration of VFSS, duration of DTI, initial CDS score, and initial MI, were analyzed using the nonparametric Mann-Whitney test. Sex, lesion side, aphasia, and neglect were analyzed by Pearson chi-square test. Mean FA and ADC values were compared between the two groups for each of the 11 ROIs. The critical value for statistical significance was set at $\mathrm{p}=0.05$. Data analyses were performed using SPSS 
ver. 17.0 (SPSS Inc., Chicago, IL, USA).

\section{RESULTS}

\section{Demographic characteristics}

A total of 31 patients with first episode MCA infarction were included (19 males, 12 females; mean age, 61.1 \pm 9.42 years). The dysphagia group included 5 women and 12 men, and the non-dysphagia group included 7 women and 7 men. Overall, the mean ages for the dysphagia and non-dysphagia groups were $62.88 \pm 9.53$ years and $58.93 \pm 9.21$ years, respectively.

\section{Clinical characteristics}

Among the 31 patients, 17 were diagnosed with dysphagia using VFSS. Right-sided lesions were observed in 13 patients (76\%) of the dysphagia group and 6 patients (43\%) of the non-dysphagia group. No group differences were observed with respect to the number of days from stroke onset to DTI, number of days from stroke onset to VFSS, Mini-Mental Status Examination, or frequency of aphasia or neglect (Table 1).

\section{DTI parameters}

The mean TVs on the ipsilesional side were 837.29 \pm $1,108.88$ in the dysphagia group and $838.21 \pm 825.84$ in the non-dysphagia group. Mean TVs on the contralesional side were $3,052.71 \pm 1,272.24$ in the dysphagia group and $2,467.29 \pm 879.53$ in the non-dysphagia group (Table 2). There were significant intergroup differences in the following four DTI values: FA values in the contra-lesional primary motor cortex $(0.41 \pm 0.04$ in the dysphagia group vs. $0.45 \pm 0.03$ in the non-dysphagia group); ADC values in the bilateral posterior limbs of the internal capsule (ipsilesional side, $0.71 \pm 0.15$ in the dysphagia group vs. $0.59 \pm 0.13$ in the non-dysphagia group; contra-lesional side, $0.64 \pm 0.05$ in the dysphagia group vs. $0.60 \pm 0.05$ in the non-dysphagia group; all $\mathrm{p}<0.05)$ (Table 3 ).

Table 1. Demographic characteristics of the dysphagia and non-dysphagia groups

\begin{tabular}{|lccc|}
\hline & Dysphagia & Non-dysphagia & p-value \\
\hline Sex (female:male) & $5: 12$ & $7: 7$ & 0.288 \\
\hline Age (yr) & $62.88 \pm 9.53$ & $58.93 \pm 9.21$ & 0.253 \\
\hline Time intervals (day) & & & \\
\hline From stroke to DTI & $44.47 \pm 21.71$ & $37.29 \pm 17.23$ & 0.257 \\
\hline From stroke to VFSS & $34.24 \pm 23.49$ & $31.07 \pm 18.75$ & 0.905 \\
\hline Lesion side (right:left) & $13: 4$ & $6: 8$ & 0.075 \\
\hline Aphasia & $5(29.41)$ & $6(42.86)$ & 0.436 \\
\hline Neglect & $7(41.18)$ & $4(28.57)$ & 0.465 \\
\hline MMSE & $18.82 \pm 8.92$ & $17.64 \pm 10.10$ & 0.735 \\
\hline Motricity index & & & \\
\hline Affected limb & $46.97 \pm 38.25$ & $35.68 \pm 30.10$ & 0.460 \\
\hline Dysphagia scale & & & \\
\hline Clinical dysphagia scale & $34.29 \pm 19.08$ & $6.36 \pm 14.27$ & $0.028^{*}$ \\
\hline
\end{tabular}

Values are presented as mean \pm standard deviation or number (\%).

DTI, diffusion tensor imaging; VFSS, videofluoroscopic swallowing study; MMSE, Mini-Mental Status Examination. Statistics were analyzed by Mann-Whitney U test or Pearson chi-square test or Fisher exact test.

${ }^{*} \mathrm{p}<0.05$.

Table 2. Comparison of volume of the corticospinal tract between the dysphagia and non-dysphagia groups

\begin{tabular}{lrrc}
\hline & \multicolumn{1}{c}{ Dysphagia } & Non-dysphagia & p-value \\
\hline Lesion side & $837.29 \pm 1,108.88$ & $838.21 \pm 825.84$ & 0.558 \\
Contra-lesional side & $3,052.71 \pm 1,272.24$ & $2,467.29 \pm 879.53$ & 0.156 \\
\hline
\end{tabular}

Values are presented as mean \pm standard deviation.

Statistics were analyzed by Mann-Whitney U test. 
Table 3. Comparison of the diffusion tensor parameters in ROIs between the dysphagia and non-dysphagia groups

\begin{tabular}{|c|c|c|c|c|c|c|}
\hline ROI & Side & Subject & ADC & p-value & FA & p-value \\
\hline \multirow[t]{4}{*}{ Primary somatosensory cortex } & Lesion & Dysphagia & $0.64 \pm 0.21$ & 0.592 & $0.35 \pm 0.09$ & 0.310 \\
\hline & & Non-dysphagia & $0.64 \pm 0.17$ & & $0.36 \pm 0.12$ & \\
\hline & Contra-lesion & Dysphagia & $0.55 \pm 0.04$ & 0.938 & $0.42 \pm 0.04$ & 0.443 \\
\hline & & Non-dysphagia & $0.55 \pm 0.06$ & & $0.43 \pm 0.06$ & \\
\hline \multirow[t]{4}{*}{ Primary motor cortex } & Lesion & Dysphagia & $0.66 \pm 0.16$ & 0.605 & $0.39 \pm 0.10$ & 0.842 \\
\hline & & Non-dysphagia & $0.63 \pm 0.05$ & & $0.42 \pm 0.04$ & \\
\hline & Contra-lesion & Dysphagia & $0.61 \pm 0.06$ & 0.369 & $0.41 \pm 0.04$ & $0.014^{*}$ \\
\hline & & Non-dysphagia & $0.61 \pm 0.04$ & & $0.45 \pm 0.03$ & \\
\hline \multirow[t]{4}{*}{ Supplementary motor cortex } & Lesion & Dysphagia & $0.67 \pm 0.16$ & 0.937 & $0.35 \pm 0.07$ & 0.827 \\
\hline & & Non-dysphagia & $0.63 \pm 0.05$ & & $0.35 \pm 0.05$ & \\
\hline & Contra-lesion & Dysphagia & $0.61 \pm 0.08$ & 0.472 & $0.39 \pm 0.09$ & 0.796 \\
\hline & & Non-dysphagia & $0.63 \pm 0.07$ & & $0.38 \pm 0.05$ & \\
\hline \multirow[t]{4}{*}{ Anterior cingulate cortex } & Lesion & Dysphagia & $0.78 \pm 0.29$ & 0.984 & $0.26 \pm 0.07$ & 0.824 \\
\hline & & Non-dysphagia & $0.70 \pm 0.08$ & & $0.27 \pm 0.05$ & \\
\hline & Contra-lesion & Dysphagia & $0.67 \pm 0.06$ & 0.953 & $0.28 \pm 0.05$ & 0.842 \\
\hline & & Non-dysphagia & $0.68 \pm 0.09$ & & $0.29 \pm 0.07$ & \\
\hline \multirow[t]{4}{*}{ Orbitofrontal cortex } & Lesion & Dysphagia & $0.74 \pm 0.44$ & 0.796 & $0.30 \pm 0.06$ & 0.539 \\
\hline & & Non-dysphagia & $0.65 \pm 0.13$ & & $0.32 \pm 0.05$ & \\
\hline & Contra-lesion & Dysphagia & $0.64 \pm 0.06$ & 0.485 & $0.32 \pm 0.03$ & 0.212 \\
\hline & & Non-dysphagia & $0.65 \pm 0.11$ & & $0.34 \pm 0.06$ & \\
\hline \multirow[t]{4}{*}{ Parieto-occipital cortex } & Lesion & Dysphagia & $0.66 \pm 0.17$ & 0.210 & $0.28 \pm 0.08$ & 0.256 \\
\hline & & Non-dysphagia & $0.69 \pm 0.15$ & & $0.25 \pm 0.09$ & \\
\hline & Contra-lesion & Dysphagia & $0.62 \pm 0.08$ & 0.535 & $0.31 \pm 0.05$ & 0.934 \\
\hline & & Non-dysphagia & $0.60 \pm 0.11$ & & $0.31 \pm 0.09$ & \\
\hline \multirow[t]{4}{*}{ Insular cortex } & Lesion & Dysphagia & $0.84 \pm 0.25$ & 0.393 & $0.23 \pm 0.08$ & 0.727 \\
\hline & & Non-dysphagia & $0.97 \pm 0.32$ & & $0.22 \pm 0.08$ & \\
\hline & Contra-lesion & Dysphagia & $0.73 \pm 0.08$ & 0.899 & $0.27 \pm 0.03$ & 0.649 \\
\hline & & Non-dysphagia & $0.73 \pm 0.09$ & & $0.26 \pm 0.04$ & \\
\hline \multirow[t]{4}{*}{ Posterior limb of the internal capsule } & Lesion & Dysphagia & $0.71 \pm 0.15$ & $0.009^{*}$ & $0.49 \pm 0.12$ & 0.368 \\
\hline & & Non-dysphagia & $0.59 \pm 0.13$ & & $0.53 \pm 0.15$ & \\
\hline & Contra-lesion & Dysphagia & $0.64 \pm 0.05$ & $0.031^{*}$ & $0.67 \pm 0.05$ & 0.309 \\
\hline & & Non-dysphagia & $0.60 \pm 0.05$ & & $0.69 \pm 0.06$ & \\
\hline \multirow[t]{4}{*}{ Thalamus } & Lesion & Dysphagia & $0.70 \pm 0.18$ & 0.136 & $0.30 \pm 0.05$ & 0.195 \\
\hline & & Non-dysphagia & $0.60 \pm 0.12$ & & $0.34 \pm 0.09$ & \\
\hline & Contra-lesion & Dysphagia & $0.70 \pm 0.09$ & 0.101 & $0.33 \pm 0.03$ & 0.102 \\
\hline & & Non-dysphagia & $0.64 \pm 0.10$ & & $0.36 \pm 0.09$ & \\
\hline \multirow[t]{4}{*}{ Basal ganglia (caudate nucleus) } & Lesion & Dysphagia & $0.75 \pm 0.34$ & 0.953 & $0.26 \pm 0.06$ & 0.613 \\
\hline & & Non-dysphagia & $0.69 \pm 0.26$ & & $0.25 \pm 0.05$ & \\
\hline & Contra-lesion & Dysphagia & $0.67 \pm 0.17$ & 0.766 & $0.28 \pm 0.07$ & 0.910 \\
\hline & & Non-dysphagia & $0.65 \pm 0.12$ & & $0.28 \pm 0.06$ & \\
\hline \multirow[t]{4}{*}{ Basal ganglia (putamen) } & Lesion & Dysphagia & $1.09 \pm 0.44$ & 0.126 & $0.23 \pm 0.08$ & 0.347 \\
\hline & & Non-dysphagia & $0.82 \pm 0.27$ & & $0.26 \pm 0.07$ & \\
\hline & Contra-lesion & Dysphagia & $0.75 \pm 0.20$ & 0.426 & $0.32 \pm 0.08$ & 0.984 \\
\hline & & Non-dysphagia & $0.69 \pm 0.12$ & & $0.32 \pm 0.05$ & \\
\hline
\end{tabular}

Values are presented as mean \pm standard deviation.

ROI, region of interest; ADC, apparent diffusion coefficient; FA, fractional anisotropy. Statistics were analyzed by Mann-Whitney U test.

${ }^{*} \mathrm{p}<0.05$. 


\section{DISCUSSION}

The present study aimed to identify the supratentorial regions related to dysphagia in MCA stroke patients, using DTI. As an extension of previous studies, we hypothesized that white matter changes after stroke are important determinants of dysphagia. In this study, dysphagia was associated with microstructural changes on either the lesional side or the contra-lesional side.

The estimated incidence of post-stroke dysphagia is $43 \%-71 \%$, and many of these cases improved within 2 weeks after stroke [2,22]. However, dysphagia persists in about $10 \%$ of the patients, who often require a nasogastric tube or gastrostomy for appropriate nutritional support $[3,22,23]$. Therefore, many researchers are trying to identify specific brain lesions associated with swallowing and factors that influence the long-term prognosis of dysphagia. Several studies have investigated the correlations between clinical findings, anatomical abnormalities, and the occurrence of dysphagia [24], but analyses of several specific brain areas have failed to identify a significant association between stroke location and the occurrence of dysphagia.

Previous studies have concluded that swallowing appears to be mediated by a distributed neural network that involves both cerebral hemispheres with descending input to the medulla. The neurophysiological and neuroanatomical functions of the supra-medullary regions are important for the control of swallowing. Through functional brain MRI, Kern identified the location of the reflexive and voluntary swallowing center in bilateral primary motor and sensory regions, insular cortex, and prefrontal lobe [25]. Hamdy et al. [26] evaluated muscle activity related swallowing using transcranial magnetic stimulation and demonstrated that swallowing centers are located bilaterally in the cerebral hemispheres, but they show interhemispheric asymmetry, independent of handedness. Other investigations for dysphagia have suggested that swallowing centers are located within and antero-caudal to the face area of the primary motor cortex [27], frontal lobe, and insular cortex [22,28]. In a recent study of stroke lesions and dysphagia, the internal capsule was most closely related to dysphagia. Furthermore, damage to the primary somatosensory cortex, motor and motor supplementary cortex, orbitofrontal cortex, and basal ganglia has been associated with dysphagia
[4]. Hence, we evaluated the above regions to investigate their association with dysphagia, and the current study showed significant differences in the FA value in the PMC on the contra-lesional side and the ADC value in the bilateral PLIC. This may be due to microstructural changes due to mirror lesions after stroke.

The role of the contralesional hemisphere in stroke recovery is controversial. Meguro et al. [29] suggested that structural changes may occur on the contralesional side because of atrophy of the corpus callosum. In addition, modifications of the electrical activity $[30,31]$, cerebral blood flow [32,33], and metabolism [32] have been reported at the cortical level, mainly within areas that mirror the stroke lesion. These findings might indicate diaschisis [30-33]. Some studies have shown increased anisotropy not only on the ipsilesional side, but also in the contralesional thalamus and cerebral hemisphere [34]. Changes in DTI values in the ipsilesional thalamus support the assumption of a loss of microscopic thalamic components because retrograde degeneration may interrupt the thalamo-cortical fibers [35]. Our findings support the notion that DTI abnormalities in these regions reflect their role in sequential movements associated with swallowing.

Cognitive function has previously been shown to be related to dysphagia. Specifically, patients with hemineglect had more dysphagia symptoms than patients with aphasia [36]. However, the current study showed no group differences in the side of the lesion, the presence of aphasia or neglect, or cognitive function.

Our study elucidated the relationship between microstructural changes in ipsilesional and contra-lesional brain regions and dysphagia in stroke patients. However, this study has significant limitations, which stem mostly from its small sample size and retrospective design. The study sample reflects only subjects with lesions in the MCA territory. Furthermore, the initial stroke volume was not analyzed. However, no significant differences were found in the corticospinal tract volume or motor function of the upper and lower limbs. Thus, we inferred that there was no significant difference in the initial stroke damage in motor function-related supratentorial regions. ROIs were hand-drawn by a rehabilitation medicine physician who had more than 5 years of experience with this technique, and every DTI parameter was measured twice and results were averaged to minimize the possible intra- 
rater reliability. In the future, we will replicate this study with a larger sample size, more sophisticated analysis, and longer follow-up.

In this study, the FA value in the primary motor cortex on the contra-lesional side and the ADC value in the bilateral PLIC showed significant inter-group differences according to the presence of dysphagia. Our finding suggests that microstructural changes in the contra-lesional hemisphere could be associated with dysphagia.

\section{CONFLICT OF INTEREST}

No potential conflict of interest relevant to this article was reported.

\section{ACKNOWLEDGMENTS}

This paper was written as part of Konkuk University's research support program for its faculty on sabbatical leave in 2014.

\section{REFERENCES}

1. Alberts MJ, Horner-Catt J. Dysphagia and aspiration syndromes. In: Bogousslavsky J, Caplan L, editors. Stroke syndrome. Cambridge: Cambridge University Press; 1995. p. 213-22.

2. Gordon C, Hewer RL, Wade DT. Dysphagia in acute stroke. Br Med J (Clin Res Ed) 1987;295:411-4.

3. Mann G, Hankey GJ, Cameron D. Swallowing function after stroke: prognosis and prognostic factors at 6 months. Stroke 1999;30:744-8.

4. Gonzalez-Fernandez M, Kleinman JT, Ky PK, Palmer JB, Hillis AE. Supratentorial regions of acute ischemia associated with clinically important swallowing disorders: a pilot study. Stroke 2008;39:3022-8.

5. Mori S, Wakana S, Nagae-Poetscher LM, Van Zijl PC. MRI atlas of human white matter. Amsterdam: Elsevier; 2005. p. 1-31.

6. Basser PJ, Jones DK. Diffusion-tensor MRI: theory, experimental design and data analysis: a technical review. NMR Biomed 2002;15:456-67.

7. Basser PJ. Inferring microstructural features and the physiological state of tissues from diffusion-weighted images. NMR Biomed 1995;8:333-44.

8. Mori S, Zhang J. Principles of diffusion tensor imaging and its applications to basic neuroscience research. Neuron 2006;51:527-39.

9. Pierpaoli C, Jezzard P, Basser PJ, Barnett A, Di Chiro G. Diffusion tensor MR imaging of the human brain. Radiology 1996;201:637-48.

10. Beaulieu C. The basis of anisotropic water diffusion in the nervous system: a technical review. NMR Biomed 2002;15:435-55.

11. Granziera C, D'Arceuil H, Zai L, Magistretti PJ, Sorensen AG, de Crespigny AJ. Long-term monitoring of post-stroke plasticity after transient cerebral ischemia in mice using in vivo and ex vivo diffusion tensor MRI. Open Neuroimag J 2007;1:10-7.

12. van der Steen JT, Ooms ME, Mehr DR, van der Wal G, Ribbe MW. Severe dementia and adverse outcomes of nursing home-acquired pneumonia: evidence for mediation by functional and pathophysiological decline. J Am Geriatr Soc 2002;50:439-48.

13. Buchholz D. Neurologic causes of dysphagia. Dysphagia 1987;1:152-6.

14. Robbins JA, Logemann JA, Kirshner HS. Swallowing and speech production in Parkinson's disease. Ann Neurol 1986;19:283-7.

15. Kim H, Na DL. Normative data on the Korean version of the Western Aphasia Battery. J Clin Exp Neuropsychol 2004;26:1011-20.

16. Schenkenberg T, Bradford DC, Ajax ET. Line bisection and unilateral visual neglect in patients with neurologic impairment. Neurology 1980;30:509-17.

17. Albert ML. A simple test of visual neglect. Neurology 1973;23:658-64.

18. Han TR, Paik NJ, Park JW, Lee EK, Park MS. Standardization of dysphagia diet. Korean J Stroke 2000;2:1915.

19. Jung SH, Lee KJ, Hong JB, Han TR. Validation of clinical dysphagia scale: based on videofluoroscopic swallowing study. J Korean Acad Rehabil Med 2005;29:34350.

20. Lee JS, Han MK, Kim SH, Kwon OK, Kim JH. Fiber tracking by diffusion tensor imaging in corticospinal tract stroke: topographical correlation with clinical symptoms. Neuroimage 2005;26:771-6.

21. Jang SH. Somatotopic arrangement and location of the corticospinal tract in the brainstem of the human brain. Yonsei Med J 2011;52:553-7.

22. Broadley S, Croser D, Cottrell J, Creevy M, Teo E, Yiu 
D, et al. Predictors of prolonged dysphagia following acute stroke. J Clin Neurosci 2003;10:300-5.

23. Carnaby G, Hankey GJ, Pizzi J. Behavioural intervention for dysphagia in acute stroke: a randomised controlled trial. Lancet Neurol 2006;5:31-7.

24. Horner J, Massey EW, Riski JE, Lathrop DL, Chase KN. Aspiration following stroke: clinical correlates and outcome. Neurology 1988;38:1359-62.

25. Kern MK, Jaradeh S, Arndorfer RC, Shaker R. Cerebral cortical representation of reflexive and volitional swallowing in humans. Am J Physiol Gastrointest Liver Physiol 2001;280:G354-60.

26. Hamdy S, Aziz Q, Rothwell JC, Singh KD, Barlow J, Hughes DG, et al. The cortical topography of human swallowing musculature in health and disease. Nat Med 1996;2:1217-24.

27. Mosier KM, Liu WC, Maldjian JA, Shah R, Modi B. Lateralization of cortical function in swallowing: a functional MR imaging study. AJNR Am J Neuroradiol 1999;20:1520-6.

28. Daniels SK, Foundas AL. The role of the insular cortex in dysphagia. Dysphagia 1997;12:146-56.

29. Meguro K, Constans JM, Courtheoux P, Theron J, Viader F, Yamadori A. Atrophy of the corpus callosum correlates with white matter lesions in patients with cerebral ischaemia. Neuroradiology 2000;42:413-9.
30. Buchkremer-Ratzmann I, August M, Hagemann G, Witte OW. Electrophysiological transcortical diaschisis after cortical photothrombosis in rat brain. Stroke 1996;27:1105-11.

31. Witte OW, Stoll G. Delayed and remote effects of focal cortical infarctions: secondary damage and reactive plasticity. Adv Neurol 1997;73:207-27.

32. Andrews RJ. Transhemispheric diaschisis: a review and comment. Stroke 1991;22:943-9.

33. Dobkin JA, Levine RL, Lagreze HL, Dulli DA, Nickles RJ, Rowe BR. Evidence for transhemispheric diaschisis in unilateral stroke. Arch Neurol 1989;46:1333-6.

34. Buffon F, Molko N, Herve D, Porcher R, Denghien I, Pappata S, et al. Longitudinal diffusion changes in cerebral hemispheres after MCA infarcts. J Cereb Blood Flow Metab 2005;25:641-50.

35. Ogawa T, Yoshida Y, Okudera T, Noguchi K, Kado H, Uemura K. Secondary thalamic degeneration after cerebral infarction in the middle cerebral artery distribution: evaluation with MR imaging. Radiology 1997; 204:255-62.

36. Schroeder MF, Daniels SK, McClain M, Corey DM, Foundas AL. Clinical and cognitive predictors of swallowing recovery in stroke. J Rehabil Res Dev 2006;43:301-10. 
Bo-Ram Kim, et al.

Appendix 1. Clinical dysphagia scale

\begin{tabular}{llr}
\hline \multicolumn{1}{c}{ Parameter } & Coded value & Score \\
\hline Location & Non-stem lesion & 0 \\
T-cannula & Stem lesion & 5 \\
& No & 0 \\
Aspiration & Yes & 25 \\
\hline Lip sealing & No & 0 \\
& Yes & 10 \\
\hline \multirow{5}{*}{ Chewing and mastication } & Intact & 0 \\
& Inadequate & 2 \\
& None & 4 \\
Tongue protrusion & Inadequate & 0 \\
& None & 4 \\
& Intact & 8 \\
\hline Laryngeal elevation & Inadequate & 0 \\
& None & 8 \\
\hline \multirow{2}{*}{ Reflex coughing } & Intact & 0 \\
\hline Total score & Inadequate & 5 \\
\hline & None & 10 \\
\hline & No & 0 \\
\hline & Yes & 30 \\
\hline
\end{tabular}

